\title{
O ensino de Física e a prática da ciência: aproximando-os com filosofia de modelos científicos no caso da Física de partículas
}

\author{
João Pedro Ghidini ${ }^{\star}$, André Fantin ${ }^{\star *}$, Ivã Gurgel ${ }^{\star \star \star}$, Marcelo Gameiro Munhoz ${ }^{\star \star \star \star}$
}

\section{Resumo}

Mundialmente, formuladores de políticas públicas, pesquisadores em educação científica, professores e alunos defendem o ensino de tópicos da Física Moderna e Contemporânea na educação básica. Dentre esses tópicos, investiga-se a potencial introdução da Física de Partículas no ensino médio. Neste contexto, há quase duas décadas, surgiram diferentes propostas críticas com relação às abordagens do tipo "Lista de Compras", que consiste em um ensino que se reduz a apresentar inúmeras partículas elementares. Neste trabalho, propomos um currículo baseado em engajamento, que busca ensinar a ciência e sobre a ciência. A argumentação sobre a ciência é vinculada às pesquisas desenvolvidas na área de Natureza da Ciência. Posteriormente, identificamos os Diagramas de Feynman como modelos a partir da concepção pragmática da Filosofia da Ciência. Por fim, a partir dessa argumentação, analisamos algumas propostas de ensino que já superaram a "Lista de Compras" vinculadas aos Diagramas de Feynman, e as comparamos a nossa proposta.

Palavra-chave: Ensino de Física de Partículas, Diagramas de Feynman, Modelo, Currículo, Natureza da Ciência.

\footnotetext{
* Mestrando em Ensino de Ciências no Programa Interunidades em Ensino de Ciências da Universidade de São Paulo. E-mail: joao.ghidini.silva@usp.br. ORCID: https://orcid.org/0000-0001-8949-6732

* Mestrando em Ensino de Ciências no Programa Interunidades em Ensino de Ciências da Universidade de São Paulo. E-mail: andre.fantin@usp.br. ORCID: https://orcid.org/0000-0002-6621-6299

*** Professor Doutor do Departamento de Física Experimental do Instituto de Física da Universidade de São Paulo. E-mail: gurgel@if.usp.br. ORCID: https://orcid.org/0000-0003-4968-6907

*.** Professor Associado do Departamento de Física Nuclear, prédio HEPIC, do Instituto de Física da Universidade de São Paulo. E-mail: munhoz@if.usp.br. ORCID: http://orcid.org/0000-0003-3695-3180
} 


\section{Introdução}

Há alguns anos que já se discutem propostas relacionadas ao ensino de Física de Partículas, tanto para a graduação em Física quanto para as escolas de ensino básico (PIETROCOLA, 2010; PASSON, ZÜGGE; GREBE-ELLIS, 2018). Por se tratar de um tópico de Física Moderna e Contemporânea, em muitos países (como por exemplo o Brasil) seu ensino nas escolas está associado à inovação curricular, o que implica numa série de dificuldades. Pietrocola e Gurgel (2017), sugerem que os obstáculos encontrados para seu ensino são tanto do tipo didático-epistemológico (relacionados às dificuldades do próprio conhecimento, como a fenomenologia, formalização, estrutura conceitual e a ontologia), quanto aos do tipo didático-pedagógico (relacionados às dificuldades do ensino desse conhecimento, associados às pré-concepções dos participantes do ambiente escolar).

Diante de tais dificuldades, mesmo nos cenários em que há o ensino de Física de Partículas (FP), alguns autores identificam problemas recorrentes nas abordagens. Na Alemanha, Passon, Zügge e Grebe-Ellis (2018) realizaram um levantamento bibliográfico sistemático da literatura europeia e anglófona de ensino de FP, incluindo defesas do conteúdo, propostas de ensino e currículos, tecendo críticas à chamada "concepção herdada" (received view) da FP. Assim é caracterizada a FP segundo esta concepção: parte-se do Modelo Padrão e apresentam-se os quarks e os léptons como "blocos fundamentais" do mundo material; caracterizam-se as interações como trocas de partículas; valoriza-se a visualização das interações, para a realização da qual, por vezes, apropria-se dos Diagramas de Feynman (DF). Nos EUA, Hobson (2011) critica essa abordagem e a nomeia "Lista de Compras" (Laundry List). No Brasil, Ostermann (2020) apontou que uma de suas preocupações é entender aspectos relacionados à Mecânica Quântica relevantes para FP pois, na ausência de discussão sobre princípios físicos dessa disciplina, no ensino médio, o tema das Partículas Elementares pode recair num exercício de classificação e memorização de um zoológico subatômico, e ainda sugeriu que a área de ensino de Física de Partículas deveria dialogar mais com as pesquisas curriculares do ensino de ciências.

Boa parte dessas preocupações mencionadas carregam como pressuposto que o conhecimento - ou mais especificamente, o conhecimento disciplinar -, tem importância fundamental para as propostas de ensino-aprendizagem. Indo além, o objetivo não é (ou ao nosso ver, não deve ser) que esse conhecimento substitua o 
conhecimento tradicional exercendo a mesma função que ele comumente exerce: decorar para ter êxito na prova ou no vestibular. Se fosse, a abordagem "Lista de Compras" não necessariamente seria um problema, sendo ainda uma boa fonte de problemas fechados para essas avaliações.

Para tentar lidar com esses problemas, este trabalho, de natureza teórica, tem os seguintes objetivos:

- Defender o ensino da Física de Partículas em um currículo baseado em engajamento.

- Identificar os Diagramas de Feynman como modelos da Física de Partículas a partir da abordagem pragmática da Filosofia da Ciência, para o ensino $d a$ e sobre a Física de Partículas.

- A partir desta perspectiva, discutir sobre as propostas que já abandonaram a abordagem da "Lista de Compras".

No restante desta seção iremos justificar porque, dado os problemas mencionados, queremos atingir os dois primeiros objetivos.

Primeiro, é importante discutir brevemente o pressuposto de que devemos nos preocupar com o conhecimento científico a ser ensinado, principalmente se levarmos em consideração a tese da Sonia Salem (2012), que aponta um movimento onde o conhecimento específico se torna implícito ou ausente na segunda metade da década de 2000 nas pesquisas da área do ensino de física no Brasil (SALEM, 2012, p. 287). Como as motivações do ensino de modelos no Ensino de Ciências (EC) por vezes partem do objetivo de ensinar sobre a ciência, essa linha a priori não pressupõe grandes comprometimentos com o conhecimento $d a$ ciência. Esse maior comprometimento com o conhecimento sobre a ciência em detrimento do conhecimento $d a$ ciência aparece explicitamente em trabalhos do levantamento de Coll, France e Taylor (2005, p. 191-192), e implicitamente em propostas que visam ensinar sobre a ciência através de conhecimentos atípicos no cânone da ciência, a exemplo da proposta de Adúriz-Bravo (2014) de utilizar narrativas.

Ao nosso ver, a identificação e o privilégio de explicitar os modelos científicos da FP durante o seu ensino não deve secundarizar o conhecimento específico dessa disciplina. Pelo contrário, esse movimento é de valorização deste conhecimento, explicitando a sua dimensão epistemológica. Gilbert (2004) aponta que o uso de modelos é uma forma de garantir um EC mais autêntico. Concordamos com essa 
afirmação, uma vez que o aprendizado genuíno da ciência é alcançado ao aprender os conceitos $d a$ ciência e sobre a ciência - e embora os modelos não sejam suficientes, eles são necessários para esse objetivo.

Há quase 30 anos atrás, Terrazzan (1992), em um breve texto, apresentava como a Física Moderna e Contemporânea (FMC) deveria ganhar mais espaço no ensino em detrimento da física clássica, tecendo argumentos que guardam premissas semelhantes às nossas, a saber: como o conhecimento da FMC pode possibilitar uma compreensão atualizada de mundo aos estudantes (e consequente melhora na participação cidadã) e ainda atualizar a forma de ensinar, uma necessidade. Essas premissas também se assemelham às justificativas que aparecem no levantamento de Ostermann e Moreira (2000), dentre outras como: atrair os jovens para carreira científica, proteção contra pseudociências, compreensão atualizada sobre o fazer científico, aproveitar-se do entusiasmo dos estudantes ao aprender conhecimentos de FMC e aumentar sua compreensão de mundo em termos de tecnologia. Na abordagem que iremos propor, algumas dessas premissas não serão o foco, pois nossa abordagem não abre tanto espaço, como justificativas para o ensino de FP, para as que defendem a atração para a carreira científica ou o entusiasmo dos estudantes. Não que elas não tenham valor algum - de fato possuem, do ponto de vista pedagógico.

Concebemos que o conhecimento da FP está imerso no interior de uma disciplina, entendendo que o conhecimento disciplinar é desenvolvido historicamente, cujo campo de ação possui compromissos epistemológicos, ontológicos e axiológicos (PIETROCOLA, 2019, p. 42). Na Teoria de Currículo, Young (2010) apresenta o que denomina como "currículo baseado em engajamento", em oposição à concepção tradicional que seria "currículo baseado em acatamento", embora ambas partam do conhecimento para elaborar o currículo. Concordamos com o autor que o currículo corresponde ao conhecimento que deve estar ao alcance de todos os estudantes, não como fatos a serem memorizados, mas explicitando sua dimensão epistemológica. Neste sentido, o currículo tem uma finalidade própria: o desenvolvimento intelectual dos estudantes, sendo que este é baseado em conceitos, e não em conteúdos ou habilidades. Devido aos conceitos serem sempre sobre alguma coisa, eles implicam alguns conteúdos e não outros. (YOUNG, 2010, p. 25). Esses conceitos são desenvolvidos no interior de comunidades de pesquisadores (YOUNG, 2010, p.25), no nosso caso, no interior da disciplina de Física. Estes conceitos permitem fazer generalizações confiáveis e são relacionados uns aos outros (YOUNG, 2010, p. 26). 
Por vezes, esses conceitos possuem referente fora da escola, como é o nosso caso (apesar de não ser um referente da vida do aluno): o DF pode ser utilizado para analisar o raio cósmico primário que interage com partículas da atmosfera. Young (2010) cita outros exemplos, como elétrons e átomos. Esses conceitos permitem que o aluno trate o mundo como "objeto de pensamento" e não como "lugar de experiência" (YOUNG, 2010, p. 25), possibilitando uma maior compreensão de mundo, baseada em um "conhecimento poderoso" (YOUNG, 2010, p. 29). Neste trabalho, o conceito central é o DF, que se relaciona com o conceito de interação de partículas, simetria e conservação. O DF é a manifestação mais popular do conceito de interação, considerando a prática científica.

Fixado o compromisso com o conhecimento, as ações pedagógicas e institucionais podem eventualmente contribuir para a resolução de problemas sociais, embora 0 problema que a escola deve se onerar a enfrentar é a desigualdade educacional. Não é, por exemplo, tarefa da escola acabar com a fome, embora a merenda escolar exerça papel importante, especialmente em regiões mais carentes. Isso também fixa um compromisso claro para a comunidade de pesquisadores em Educação Científica que concordarem com esses pressupostos e buscarem realizar intervenções: o conhecimento a ser lecionado tem papel importante (embora, ressaltamos, não seja a única preocupação). Isso contribui para a diminuição da desigualdade educacional a nível nacional (especialmente em trabalhos com escolas públicas) e também a nível internacional (especialmente para países em desenvolvimento).

Dada a complexidade do conhecimento da FP (manifestada por exemplo pelos obstáculos didático-epistemológicos citados), para que se torne viável seu ensino em sala de aula, é necessária a realização da transposição desse conhecimento. $\mathrm{Na}$ perspectiva deste trabalho, o desafio está em aproximar o máximo possível os modelos científicos a serem ensinados da episteme dos modelos científicos da comunidade científica.

Apesar disso, a diversidade de disciplinas meta-científicas indica que explicitar a dimensão epistemológica é um desafio. De forma mais específica, esse problema se situa em responder: o que da dimensão epistemológica dos DF nós queremos explicitar para engajar os estudantes neste modo de tomar o mundo como objeto de conhecimento? Podemos encontrar respostas para essa pergunta através da grande área de pesquisa em Educação Científica nomeada como Natureza da Ciência (NdC). Em diferentes literaturas é possível encontrar a afirmação de que a definição 
de o que é NdC não é consensual. Moura (2014, p. 37) em seu levantamento sugere a seguinte resposta (ainda que reconheça que é limitada): "estudar a natureza da Ciência significa compreender como o homem constrói o conhecimento científico em cada contexto e em cada época, tendo como base suas concepções filosóficas, ideológicas e metodológicas”.

As pesquisas realizadas em $\mathrm{NdC}$, sendo uma área de pesquisa de longa data, se espalham em diferentes propósitos, como: Por que ensinar, O que ensinar, Como ensinar e Instrumentos para avaliar as concepções de $\mathrm{NdC}$ de estudantes e professores. Lederman (2007) realizou um ótimo trabalho de revisão sobre as diferentes pesquisas da área até então.

Em uma dimensão de Por que ensinar, Lederman (2007), em seu balanço sobre a área, pontua que a NdC é um importante componente para a Alfabetização Científica. Driver et al. (1996) estabelece cinco linhas argumentativas (Econômica, Utilitarista, Democrática, Cultural e Moral). Diferentes argumentos dessas linhas argumentativas e diferentes autores sugerem que o aprendizado da NdC estaria associado à melhora da cidadania. Neste contexto, Hodson (2018) busca aprofundar a discussão sobre que cidadania estamos falando, defendendo um currículo que forme um cidadão científico radical (HODSON, 2018, p. 46).

Como a discussão aqui estabelecida foi partindo do conhecimento $d a$ ciência, e não do conhecimento sobre a ciência, nós não nos aprofundaremos na discussão sobre Por que ensinar a NdC na Educação Científica. Gostaríamos de pontuar apenas que, concordando com Pereira e Gurgel (2020), é um desafio do nosso tempo evitar o "positivismo ingênuo" e, ao mesmo tempo, evitar o relativismo epistêmico. Neste sentido, a explicitação da dimensão epistemológica tomando o conhecimento científico a partir da abordagem de modelos na perspectiva pragmática é nossa aposta. Por outro lado, como a NdC, de certo modo, estará presente na nossa proposta, é natural que alguns objetivos da $\mathrm{NdC}$ sejam cumpridos - exceto aqueles objetivos que possuem um resultado final bem especificado, como no caso de Hodson (2018).

Na dimensão de $\mathbf{O}$ que Ensinar, convergindo com Irzik e Nola (2011, p.593), concebemos que a separação da Investigação Científica (IC) da NdC, como proposta por Lederman (2007; 2018), é meramente artificial, uma vez que os elementos que compõe a investigação são por si só elementos da $\mathrm{NdC}$ e a IC é parte importante para entender as características epistemológicas sugeridas por autores da "Visão Consensual”, que descrevem essas características da ciência organizada em itens 
(tenets), através de poucas linhas (KIMBALL, 1967) ou um parágrafo (LEDERMAN, 2007). Nesse sentido, concebemos os modelos como parte importante do ensino da NdC, algo que não é de fato novo na literatura dessa área (IRZIK; NOLA, 2011; DUSCHL; GRANDY, 2013; SANTOS; MAIA; JUSTI, 2020; MARTINS, 2015).

Ariza, Lorenzano e Adúriz-Bravo (2016), apontam que os avanços na filosofia da ciência têm sido excluídos das propostas didáticas, apesar de uma parcela de pesquisadores aderirem à abordagem de modelos - mais especificamente, à abordagem semântica. Neste trabalho, os autores apresentam quatro abordagens da família semântica: Ronald Giere, Bas Van Fraassen, Frederick Suppe e o Estruturalismo meta-teórico. Eles também apontam que há uma simplificação exagerada da ideia de modelos (dentre outras ideias meta-teóricas) nas apropriações da área de EC e, portanto, buscam apresentar os pontos principais das abordagens semânticas, de modo que haja uma apropriação mais convergente dessas ideias meta-teóricas com o uso das mesmas nas disciplinas meta-científicas. Concordamos com esse diagnóstico, porém neste trabalho partimos não da abordagem semântica, mas da pragmática, cujas distinções serão esclarecidas na próxima seção.

Em resumo, defendemos um currículo baseado em engajamento, o que implica explicitar a dimensão epistemológica dos conceitos, ao mesmo tempo em que este conceito tem papel central. Uma forma de explicitar a dimensão epistemológica é a partir do conceito de modelos - mais especificamente, tomando os DF como modelos. Este é um movimento contrário à "Lista de Compras". Modelos têm um papel de destaque na $\mathrm{NdC}$, especialmente quando consideramos a investigação e a natureza do conhecimento produzido.

Por outro lado, os conhecimentos mais seguros estão localizados no interior de disciplinas. Deste modo, os conhecimentos mais seguros sobre a ciência se localizam no interior das disciplinas meta-científicas. Sendo assim, para encontrarmos uma melhor caracterização de modelos devemos voltar-nos à Filosofia da Ciência. O que desta caracterização será útil para a sala de aula é um segundo movimento a ser realizado. A escolha pela abordagem pragmática de modelos se dá pois acreditamos que ela explicita melhor os problemas da concepção herdada do ensino de Física de Partículas, isto é, a baixa correlação entre o papel dos DF na sala de aula e na prática científica. 


\section{Modelos Científicos e os Diagramas de Feynman}

Apesar de alguma centelha de interesse em seus primórdios, por parte de grandes nomes como Ernest Nagel e Norman Campbell, a filosofia da ciência profissional voltou-se ao papel dos modelos na investigação científica há muito pouco tempo, por volta da segunda metade do século XX. Esse movimento esteve inicialmente associado a uma troca de paradigma sobre a concepção de teoria científica, do sintático (que compôs a chamada Visão Recebida da Filosofia da Ciência), para o semântico ${ }^{1}$ (VAN FRAASSEN, 1991, p. 1-17). Enquanto no primeiro elas são entendidas como um conjunto de axiomas das quais deduções verdadeiras são tiradas sintaticamente, usando os métodos da lógica, na segunda elas são entendidas como modelos semânticos de alguma estrutura lógica pré-definida. Nessa segunda concepção, o significado atribuído aos termos e aos predicados da teoria são tão essenciais para avaliar a validade de suas conclusões quanto a sintaxe da lógica em que se escreve a teoria.

Entretanto, a adoção do termo "modelo" da lógica acaba causando, ironicamente, uma confusão semântica com o uso tradicional do termo "modelo" por cientistas, esse referindo-se a um conjunto de objetos que servem de ferramentas auxiliares em uma investigação, na qual está geralmente envolvida alguma abstração ou idealização, junto aos experimentos e às teorias, mas sem reduzir-se a nenhum dos dois. $\mathrm{O}$ filósofo da ciência Luís Henrique de Araújo Dutra estudou em seu livro Pragmática de Modelos o papel desses objetos na ciência, ressaltando a autonomia desse significado de modelo em relação àquele que de certa forma domina a filosofia da ciência contemporânea, o semântico, distinguindo o primeiro pelo epíteto de "científico"2.

Os modelos científicos seriam, então, classificados como nômicos, a maneira como Nagel e Campbell os compreenderam, quando codificam algum enunciado nomológico. Essa lei, expressa de maneira completa ou aproximada em algum sistema-modelo é então abstraída das características contingentes do sistema-modelo e usada em novas investigações, realizadas em sistemas agora chamados de modelados, como uma primeira aproximação de seu comportamento, como um teste da teoria, como uma simulação, etc. Um exemplo da Física para esse tipo de modelo é a Lei de Ohm, que relaciona linearmente a corrente elétrica com um diferença de potencial em um circuito, essencialmente a mesma que a Lei de Poiseuille, que relaciona linearmente o volume de fluido que atravessa uma certa seção transversal dada uma diferença de pressão (DUTRA, 2013, p. 93-94). Não há um sentido 
absoluto em que um sistema é modelo ou modelado. No caso citado, portanto, não há diferença essencial entre dizer que o circuito elétrico é modelado por um cano ou que um cano é modelado por um circuito elétrico ${ }^{3}$.

Um tipo de modelo científico próximo ao nômico, porém mais amplo, é o matemático. Entendemos esse tipo de modelagem como aquela por trás de boa parte das ciências naturais - em particular da Física - e algumas ciências humanas, em que a certas características e relações do mundo concreto corresponde-se certas entidades e relações matemáticas as quais podem, fazendo uso de suas propriedades intrínsecas, informar propriedades, relações e comportamentos do mundo concreto previamente insuspeitas. Nesse tipo de relação entre modelo e modelado existe uma certa assimetria, com as propriedades do sistema modelo sendo uma fonte muito mais importante de informação sobre o sistema modelado que esse último sobre aquele.

A importância desse tipo de assimetrias foi ressaltada pela filósofa Mary Hesse, dando como exemplo de modelo o gás ideal (DUTRA, 2013, p. 51-52). Comparamos, nesse modelo, as partículas de um gás a bolas de bilhar. Fazemos isso supondo que essas duas classes de objetos possuem uma série de propriedades em comum, que aqui resumimos como aquelas que caracterizam um ponto material newtoniano, as quais permitem essa aproximação. Hesse chamará essa a analogia positiva do modelo. Existem uma série de outras características que não sabemos se são comuns aos dois sistemas, é o aspecto neutro da analogia, e é nesse campo que o modelo pode fornecer conhecimento sobre o modelado, através do teste experimental e da simulação. Existe, ainda, a analogia negativa, os sentidos em que os sistemas são dissimilares. É na delimitação dessas analogias que se encontra a assimetria entre os dois sistemas, de natureza epistemológica, pois há, na prática científica, um sentido claro em que um sistema é modelo e o outro é modelado: é quando sabe-se mais sobre um sistema do que sobre o outro. É justamente fazendo uso de um sistema mais conhecido, modelo, e das suas analogias positivas com um sistema menos conhecido, modelado, que se empreende a atividade de modelagem, de forma a testar o campo de possibilidades das analogias neutras.

Essa abordagem de se identificar e compreender a natureza de elementos da ciência, no caso os modelos científicos, a partir de seus usos e de sua dinâmica na prática científica é chamada na filosofia da ciência de pragmática e é ela que nos interessa no presente trabalho. 
Mencionaremos agora duas importantes compreensões pragmáticas de modelo científico. A primeira é o conceito de máquina nomológica de Nancy Cartwright (DUTRA, 2013, p. 130-159; CARTWRIGHT, 1999, p. 49-75). Máquinas nomológicas seriam sistemas concretos elaborados de maneira que exibem um comportamento expressado diretamente por uma lei científica ${ }^{4}$, sem a confusão de efeitos cruzados e causas múltiplas da maioria dos sistemas mais realistas. Um projeto de máquina nomológica seria o sistema abstrato correspondente à máquina nomológica, que é um modelo científico. Um exemplo da Física Aplicada de máquina nomológica é o laser, cujo projeto de máquina nomológica correspondente seria, dentre vários possíveis, o simplificado modelo de três níveis presente em muitos livros didáticos (EISBERG; RESNICK, 1985, p. 397; CARTWRIGHT, 1983, p. 148). Para Cartwright, a prática científica seria centralizada em torno desses sistemas, que, expressamente elaborados para encaixar-se nos conceitos e leis da teoria, seriam usados para testá-las, desenvolver aplicações, planejar experimentos, computar simulações e, o mais importante, possibilitar conhecimento acerca de sistemas que, a princípio, não seriam alcançados pela teoria.

Dutra (2013) defende que, justamente pelas máquinas nomológicas terem essas características, elas seriam tão modelos quanto seus projetos, ainda que modelos concretos, no caso. Isso conecta a concepção de máquina nomológica com a segunda compreensão que gostaríamos de destacar, a de modelos como mediadores entre teoria, experimento e o mundo concreto, elaborado pelas filósofas Margaret Morrison e Mary Morgan (MeM).

É central para essa concepção que os modelos científicos sejam entidades autônomas em relação aos domínios concreto (que se quer estudar) e ao da teoria. Essa autonomia concede aos modelos, por serem, fundamentalmente, ferramentas adicionais às teorias no repertório da ciência, sua capacidade de representar os fenômenos do mundo concreto, transcendendo as limitações das teorias. A noção de representação considerada pelas autoras foge à noção intuitiva de "semelhança" física (no caso de modelos concretos) ou isomórfica (no caso de modelos abstratos) com o mundo concreto, estando mais próxima de um sentido epistêmico: a representação oferecida pelo modelo consiste no que pode-se aprender sobre o aspecto do mundo modelado por ele. Assim, os modelos seriam representantes dos sistemas modelados.

Sendo a representação, nessa concepção, em sentido epistêmico, é na análise da etapa de construção e aplicação do modelo que se compreende o conhecimento 
alcançado com ele, em lugar de uma análise in vacuo, que seria característica da concepção semântica de representação.

Um exemplo característico da concepção de modelo das autoras é o túnel de água de Prandtl, usado para entender a natureza da interação de um fluido com um objeto sólido (MORRISON; MORGAN, 1999, p. 26-27). Esse modelo permitiu a Prandtl identificar, visualmente, duas regiões de fluxo do fluido sobre um objeto disposto no túnel. O uso desse modelo concreto ensinou a Prandtl um conhecimento que ele tomou em seguida como base para uma modelização abstrata, matematizada.

Observa-se que o túnel de água funcionou como um representante de situações mais realistas de interação fluido-sólido por conduzir a conhecimento válido acerca da situação modelada. Conhecimento este obtido pois elementos experimentais, teóricos e externos a ambas foram utilizados para construir o modelo, e porque sua construção e manipulação informou o uso da teoria no tratamento do problema. Exemplos análogos podem ser encontrados na fabricação de lentes, no estudo da estabilidade nuclear e em experimentos com laser (MORRISON \& MORGAN, 1999, p. 23-25).

A seguir, faremos uma breve introdução aos diagramas de Feynman, ressaltando os elementos que ajudarão a explicá-los como modelos no sentido de MeM.

Os diagramas foram introduzidos pela primeira vez na Física no encontro de Pocono, em 1948, no contexto do "problema dos infinitos" da Eletrodinâmica Quântica (QED), consistindo essencialmente em divergências que apareciam no cálculo da alteração da energia do elétron em um processo de espalhamento (ou seja, perturbativo) entre dois elétrons. Processos complicados de renormalização, em que as divergências são engenhosamente assimiladas no cálculo através da alteração do valor da massa e da energia dos elétrons em interação, foram desenvolvidos por Julian Schwinger e Sin-Itiro Tomonaga ${ }^{5}$, mas eram pouco disseminados para além de grupos em interação direta com seus autores, até Pocono.

Nesse encontro, Feynman introduziu com o primeiro diagrama, de um espalhamento elétron-elétron, uma maneira iconográfica engenhosa de "tomar nota" de todos os elementos da matriz de espalhamento (Matriz-S) com contribuição importante para o cálculo das energias envolvidas usando o procedimento de renormalização. Após o encontro, foi estudado, desenvolvido e disseminado por Freeman Dyson, e o método de cálculo de perturbações fazendo uso de diagramas de Feynman espalhou-se para além dos limites da QED e mesmo da Física de Partículas, sendo usados 
em outras teorias de campo quântico, como a Teoria de Mésons, a Cromodinâmica Quântica, e em outras disciplinas, como a Física da Matéria Condensada. Esse sucesso foi em parte atribuído aos diagramas relacionarem-se a uma certa tradição de representação iconográfica na Física que data da introdução dos diagramas de espaço-tempo de Minkowski, décadas antes, e pela semelhança com trajetórias em câmaras de nuvens (KAISER, 2005).

Apesar de ser essencialmente um instrumento para o cálculo da Matriz-S, e de apenas o produto interno da série ter sentido físico ${ }^{6}$, os Diagramas de Feynman (DF) desde sua introdução são apropriados como uma representação icônica dos processos que representam na linguagem de uma teoria física essencialmente não-icônica, a Mecânica Quântica (MQ), na qual não devemos falar fundamentalmente de trajetórias de partículas. Na próxima sessão iremos, com Passon, Zügge e Grebe-Ellis (2018), apresentar exemplos de como essa concepção dos DF permeia os usos desse recurso no ensino da FP. Por enquanto, apresentaremos a leitura de Stöltzner (2017) dos DF como mediadores (MeM).

A primeira característica de um modelo como mediador atendida pelos DF é a autonomia, como a referência nos parágrafos anteriores ao seu uso em teorias e disciplinas bastante diferentes para a qual foi concebido (QED). Os DF também servem como representantes no sentido de MeM: em um espalhamento elétron-elétron, por exemplo, o DF correspondente pode ser tomado como representante de toda a série infinita contida na Matriz-S7, assim como cada "ramo" (cada linha traçada do diagrama) pode ser tomado como representante de um termo diferente do cálculo perturbativo.

Podemos também desenhar um DF apenas com os ramos que consideramos mais importantes em certo processo e tomá-lo como representante do processo inteiro, como é o caso da guia de produção do bóson de Higgs dada pela fusão de glúons (Figura 1), que ajuda a explicar a obtenção da partícula, assim como a orientar a procura experimental por ela. Esse procedimento, então, envolve uma reordenação da série visando um objetivo explicativo e preditivo, e por isso é modelização no sentido de $\mathrm{MeM}^{8}$. 
Figura 1: O diagrama de Feynman representando a guia de produção do bóson de Higgs.

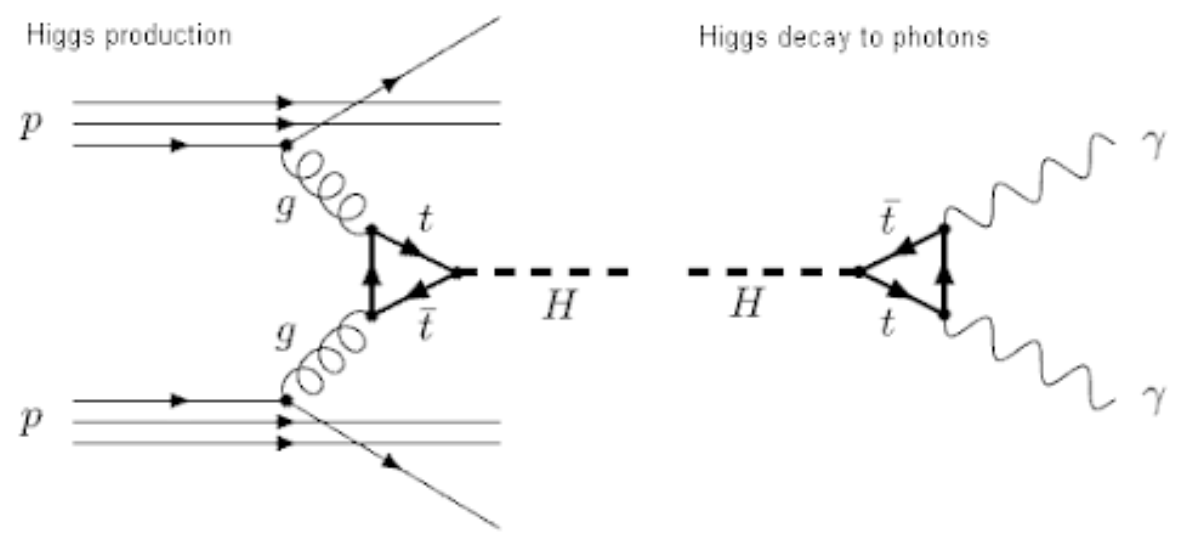

Fonte: http://lppp.lancs.ac.uk/higgs/en-GB/higgs.html?LPPPSession=1567036800030.

Em um contexto similar, mas agora no domínio da espectroscopia, podemos desenhar apenas os ramos do DF (ou tabelar as ordens do cálculo perturbativo) correspondentes aos termos contabilizados à luz da precisão da mensuração realizada, por exemplo do Desvio Lamb das linhas espectrais do Hidrogênio. Esse exemplo ilustra o papel de simulação e planejamento de experimentos que os modelos, na perspectiva de MeM, promoveriam.

Vemos, nessa breve exposição, como os DF, devido à sua flexibilidade e utilidade para os mais diversos objetivos da prática científica, enquadram-se, no entendimento da vertente pragmática da filosofia da ciência, como modelos científicos. Na próxima seção avaliamos como os DF são usualmente transpostos para a prática educacional e como aparecem na pesquisa acadêmica em ensino de FP, destacando os sentidos em que nossa proposta, partindo das perspectivas educacional e filosófica delineadas no artigo, afasta-se daquelas.

\section{Os Diagramas de Feynman na literatura de Ensino de Ciências e na prática pedagógica}

Passon, Zügge e Grebe-Ellis (2018) fizeram um levantamento da presença de FP em currículos escolares, geralmente ocupando quase que integralmente o módulo reservado à Física Moderna e Contemporânea, e na literatura de ensino de ciências. Perceberam que as abordagens alternativas à FP defendidas por autores da área de 
ensino que opõem-se à "Lista de Compras" incluem diversos equívocos de natureza conceitual e interpretativa, os dois principais sendo: a escolha de uma interpretação ontológica de uma teoria quântica de campos (TQC) sem "aviso de precaução", e a natureza representacional dos Diagramas de Feynman.

A interpretação ontológica mais disseminada da TQC, ou seja, a resposta mais comum a pergunta de a que entidades essa teoria se refere e descreve, segue a “concepção herdada” em admitir que são "partículas elementares”, sendo portanto uma interpretação “corpuscular” (ALLDAY, 1997; FARMELO, 1992; JOHANSSON; WATKINS, 2013) $)^{9}$ Um dos problemas com essa abordagem é que ela não consegue explicar a impossibilidade de localização dessas entidades e suas interações em termos da teoria (atribuir autofunções/autoestados de posição).

Autores que defendem uma interpretação “ondulatória” da TQC (DANIEL, 2006; HOBSON, 2005; 2013) atribuem realidade a uma ou mais de uma espécie de campo "universal" preenchendo o Universo, capaz de ser "excitado", e cujas “excitações" teriam papel importante nas interações medidas nos experimentos e na descrição teórica dessas interações.. O principal problema que cerca essa abordagem segue de o elemento teórico de uma TQC que assume o papel de "campo" de uma teoria clássica serem operadores da MQ, entidades que têm existência interdependente do vetor de estado/função de onda. Assim, responder "o campo" à pergunta "a que entidade a TQC se refere?" Exige uma segunda pergunta: “em que entidade se aplica o campo?".

Os DF, por sua vez, costumam aparecer quando a tentação de usar ferramentas tradicionais do ensino de física (clássica), como visualizações e imagens mentais, supera a cautela dos autores, em particular porque passa por outra tentação do ensino de física: evitar a matemática (WOITHE; WIENER; VAN DER VEKEN, 2017). Há os autores que reproduzem a visão mais ingênua dos DF, de que são representações visuais de eventos espaço-temporais, ou de que representam processos físicos ocorrendo "na realidade" (JONES, 2002; KONTOKOSTAS; KALKANIS, 2013) ${ }^{10}$, mas há também os autores que, em uma tentativa de conciliar o uso didático dos DF com o mínimo de discussão técnica da sua natureza, confundem interpretações, além de cometer erros conceituais, e chegam a conclusões equivocadas (ALLDAY, 1997; ORGANTINI, 2011).

Allday (1997) busca discutir a "natureza da força na física de partículas". O autor recheia a discussão com suas experiências na formação de professores e com alunos, 
exemplificando esses conceitos principalmente através de analogias. Dentre as analogias, ao discutir sobre a atração e a repulsão, o autor compara os diversos termos da série representados nos ramos de um DF aos diversos movimentos simultâneos que um ponto material pode desenvolver na Mecânica. Essa interpretação dos DF, entretanto, além de seguir em direção contrária à interpretação majoritariamente corpuscular do autor (assemelha-se ao que a interpretação ondulatória da MQ diria sobre um experimento de dupla fenda), é conceitualmente equivocado, porque ignora que se toma o produto interno da série (os processos têm de "interferir" entre si, como na MQ).

Além disso, Allday (1997) apresenta majoritariamente características gerais dos DF, e pouco diz sobre os usos desses dispositivos na prática científica. Organtini (2011) usa o argumento do sucesso preditivo para defender a realidade dos processos descritos pelos DF. Esse argumento, entretanto, só tem valor no contexto do debate, interno à disciplina de Filosofia da Ciência, acerca da realidade de entidades inobserváveis em teorias, o que não é o caso dos DF.

Já Dunne (2001, p. 367, tradução nossa) aponta: “O objetivo deste artigo é uma tentativa de persuadir a comunidade de ensino a tomar conhecimento das regras de construção dos diagramas e adotar uma abordagem consistente para a representação das reações das partículas”. A construção argumentativa do autor sugere que os DF seriam representações das interações entre partículas, apontando ainda que: "A descrição das interações em palavras ou equações faz com que o assunto pareça muito complicado e difícil de acompanhar. O uso de diagramas de Feynman torna muito mais fácil categorizar e visualizar o que está acontecendo" (p. 369, tradução nossa).

Quanto aos sentidos de representação, o autor toma o cuidado de apontar que os vértices não representam as trajetórias espaço-temporais das partículas. $\mathrm{O}$ artigo é repleto de diferentes DF, servindo de exemplos para que o autor explicite as regras de sua construção. No final do artigo, o autor esclarece que esse é um movimento de mostrar os DF mais como uma "ferramenta formal" ao invés de uma "ilustração informal". Sob a luz da nossa discussão, esse trabalho valoriza mais os DF em termos de "habilidade" (aprender corretamente as regras para elaborar os DF). O autor traz poucos esclarecimentos sobre o que está sendo representado nos $\mathrm{DF}$, e em alguns trechos (como o citado acima), seu artigo pode levar a um equívoco conceitual. Tomando a noção de representante de que estamos nos apropriando, a partir da pragmática de modelos, o DF é representante de toda a série infinita 
contida na Matriz-S. Em termos de prática científica, os DF estão intimamente ligados à matematização: mais próximos de um sistema de equações que de um diagrama de corpo livre.

Ainda que tomemos o sentido de representação de forma pouco restrita, com um sentido próximo à linguagem natural, a utilização da representação visual que se propõe a partir dos DF pode ser explorada como um recurso pedagógico, mas sem ignorar sua representação epistêmica (como representante, no sentido de MeM). Do contrário, o conceito sofre grande descaracterização.

A utilização da representação visual como um recurso pedagógico aparece com mais intensidade em Pascolini e Pietroni (2002). Os autores, buscando evitar os problemas conceituais que surgem quando se impõe as imagens do mundo macroscópico no mundo microscópico, afirmam que: "Em vez disso, decidimos explorar as imagens geradas pela matemática da teoria quântica de campos, ou seja, os diagramas de Feynman, que pensamos que poderiam desempenhar o papel de metáforas precisas" (p. 325, tradução nossa).

Com isso, os autores sugerem que os estudantes podem aprender diversas conceitos, como: "[...] a relação entre matéria e antimatéria, a indistinguibilidade de partículas idênticas, a existência de partículas virtuais e seu papel como mediadores de interações, e assim por diante" (PASCOLINI; PIETRONI, 2002, p. 325, tradução nossa).

Desse modo, o conceito DF acaba sendo um meio para estudar outros conceitos. Eles relatam uma atividade ministrada para estudantes, que consiste na construção de modelos mecânicos dos DF, utilizando materiais como latas e varetas (PASCOLINI; PIETRONI, 2002, p.326). Dadas as restrições mecânicas, não é possível "montar" um DF que viole as leis de conservação. Apesar de, em princípio, isso trazer dúvidas se os estudantes efetivamente estarão compreendendo esses outros conceitos (como o de conservação) ou se na verdade estão apenas realizando um trabalho manual - sendo que essa última é uma interpretação que é corroborada pelo próprio relato dos autores, em que os estudantes tentaram por diferentes meios construir um DF que era impossível e apenas poucos notaram que era impossível -, os autores indicam que a utilização dessa atividade melhorou o desempenho dos estudantes ao lidarem com questões envolvendo os DF sem esse sistema mecânico, especialmente para questões envolvendo DF que eram impossíveis. 
Não é claro o que efetivamente é discutido com os estudantes sobre Diagramas de Feynman, embora os autores mencionem realizarem uma introdução aos fenômenos descritos pela QED (PASCOLINI, PIETRONI, 2002, p. 327). Novamente, a utilização de recursos imagéticos e mecânicos como instrumentos pedagógicos não se configuram, a priori, como um problema, mas se tornam na medida que em suas limitações não são explicitadas. Por outro lado, tomando os DF como modelos, eles possuem uma finalidade específica na investigação científica - que, majoritariamente, não é a que os autores estão propondo. Esse é um aspecto que poderia ser explicitado na proposta, mas que não foi, pois, os autores não encaram esse conceito dessa forma.

Outros dois interessantes trabalhos, que estão correlacionados, são Hoekzema et al. (2005) e Van der Berg e Hoekzema (2006). No primeiro trabalho, Hoekzema et al. (2005) apresenta uma parte do projeto de ensino de FMC - mais especificamente o texto para estudantes sobre transformação, equação da reação, leis de conservação e simetria. As leis de conservação e simetria, que são o foco de Van der Berg e Hoekzema (2006), são ensinadas utilizando diagramas de reação (expressão utilizada no texto para estudantes) ou diagramas de Feynman simplificados (VAN DER BERG; HOEKZEMA, p. 48, 2006). Conforme Hoekzema et al. (2005, p. 266), a preferência por utilizar diagramas de Feynman simplificados (que são usados apenas para descrever e prever reações, sem inferir a probabilidade de reações ou examinar mais profundamente a natureza das interações) se deu devido à uma experiência anterior com os DF, que não foi bem-sucedida.

Os autores ressaltam que os diagramas de reação foram bem recebidos pelos professores, sendo muito mais 'compreensíveis', e os autores suspeitam que os professores acham tão fácil que 'vão rápido', com poucos exercícios (HOEKZEMA, 2005, p. 271). No texto do aluno, os diagramas de reações aparecem como uma forma de 'visualizar as reações', em que o foco principal é como as leis de conservação são manifestadas e permitem realizar operações. Essa relação com o conhecimento é mais explícita no artigo de Van der Berg e Hoekzema (2006). Neste artigo, os autores relatam a utilização de um método de rápido feedback, que consiste em um método de ensino em que o professor dá uma série de tarefas curtas para serem realizadas individualmente, mas de forma coletiva (VAN DER BERG; HOEKZEMA, 2006, p. 49). Contrastando esse projeto com o nosso trabalho, diferentes pontos podem ser discutidos. Primeiro, que a relação que os autores tecem com os DF é análoga a de Pascolini e Pietroni (2002): os DF são um meio para entender algum outro conceito. 
Como já dissemos, tomando os DF como modelos, o que deve ser ressaltado muda. Um segundo ponto, embora os trabalhos estejam ensinando conceitos, o ensino de conceitos está mais baseado em um 'currículo baseado em acatamento' do que um 'currículo baseado em engajamento'. Isso porque a base epistemológica desses conceitos não é explicitada. A relação estabelecida com os conceitos é operacional (o que a priori não é um problema), mas somente operacional.

Terceiro, a utilização de diagramas de reações ao invés de DF é uma separação artificial. Possui a boa intenção de tornar a prática didática mais simples e ao mesmo tempo não falar explicitamente de DF, pois DF é um outro conceito que será estudado na universidade. Além de ser contestável se efetivamente a turma seguirá carreira científica, utilizando os diagramas de reações, permanece uma lacuna conceitual dos DF que será preenchida pelas concepções espontâneas dos próprios estudantes.

\section{Considerações finais}

Vemos como, apesar de muitos autores da área de Ensino de Ciências concordarem que o ensino de FP é seriamente falho dado a disseminação da "concepção herdada" do Modelo Padrão e da "Lista de Compras", não há consenso de como operar a transposição desse conteúdo. Em meio a esse dissenso há, inclusive, propostas que incluem equívocos conceituais, como a maior parte daquelas que se apropriam dos DF, uma vez que eles prestam-se, em uma interpretação que ignora seu uso na prática científica, a serem tomados como recursos pedagógicos que adaptam-se a características muito arraigadas da cultura escolar mais ampla, e da específica de ensino de física, majoritariamente de física clássica (GURGEL; PIETROCOLA, 2017): a ênfase em imagens, intuições e pouca matematização, ou uma matematização que reduz-se a um mero "formulismo", termo célebre cunhado por Zanetic (1989).

Concluímos que se a FP for figurar no currículo da escola básica, é preciso que esse conhecimento sofra menos distorções quanto possível, e por isso, é preciso que professores sejam formados com uma versão transposta desse conhecimento que melhor reflita as práticas que este estrutura, e a episteme que, por sua vez, o estrutura. Em outras palavras, conhecimentos de FMC só entrarão na escola básica se a formação dos docentes promover autonomia em relação a esses saberes, e apostamos que essa autonomia só será alcançada através de um ensino que convirja com a 
prática científica. Esse aspecto é crucial para o senso de identidade dos professores como membros de uma profissão (YOUNG, 2010, p. 27).

Em nosso esboço de proposta pedagógica para o ensino de FP, mergulhamos, portanto, nas características epistemológicas específicas dessa disciplina e de sua prática e não demos como resolvidos problemas em aberto de interpretação das teorias envolvidas. Através desse mergulho, pudemos identificar elementos desse saber que nos permitem simultaneamente valorizar a natureza do conhecimento de FP e o conhecimento característico da disciplina, convergindo com nossas afiliações teóricas e axiológicas da Teoria de Currículo e da Educação Científica. Por consequência, não apresentamos fórmulas prontas nem uma proposta acabada, mas abrimos o caminho para que futuras propostas seguindo essas diretrizes não tropecem em velhos problemas. Gostamos de pensar que, ao invés de engajar-nos com os problemas "normais" do ensino de FP, estamos propondo uma nova perspectiva a partir da qual esses problemas são entendidos de maneira diferente, e em que, portanto, novos tipos de soluções são possíveis, abrindo um horizonte ainda a ser perscrutado.

\section{The teaching of physics and the practice of science: bridging them with the philosophy of scientific models in the case of particle physics}

\section{Abstract}

Globally, public policy formulators, science education researchers, teachers and students sustain the teaching of topics from Modern and Contemporary Physics in basic school. Among those topics, the potential insertion of Particle Physics in secondary school is investigated. In this context, already present in publications from over two decades, are critical propositions to the "Laundry List", which consists in a teaching that is reduced to presenting the numerous elementary particles, much criticized for promoting a "received view" of the Standard Model. In the present work, converging with this criticisms, we propose an engagement based curriculum that seeks to teach the content of science and about science. The argumentation about science is connected to investigations developed by the Nature of Science area. Afterward, we identify the Feynman Diagrams as models in the understanding of the pragmatic conception of the Philosophy of Science. Finally, we analyse some pedagogical proposals involving the Feynman Diagrams that have overcome the "Laundry List" approach and compare them to our proposal.

Keywords: Particle Physics Teaching, Feynman Diagrams, Models, Curriculum, Nature of Science. 


\section{Notas}

1 Estamos nos restringindo, nesse breve histórico, à filosofia da ciência de tradição analítica, centrada em países anglófonos, e derivada dos trabalhos de Bertrand Russel, do primeiro Wittgenstein e, mais tarde, de David Lewis, no começo do século XX. Daí a centralidade dessa leitura lógico-linguística da ciência.

2 Apropriamo-nos mais das discussões epistemológicas do livro, deixando um tanto de lado a discussão metafísica acerca do estatuto existencial dos modelos. Embora, como veremos, ela possa contribuir para a discussão acerca da existência das entidades de que trata a Física de Partículas, que é relevante para seu ensino, a evitamos no presente trabalho, deixando-a para o futuro.

3 A análise pretende-se, portanto, válida qualquer que seja a perspectiva do cientista. Assim, ela faz-se útil tanto para um estudante de Física que aprende Mecânica dos Fluidos depois de Eletromagnetismo quanto para um estudante de História da Física, para quem o sentido de modelo e modelado será invertido.

4 Na preferência de Cartwright (1999) diríamos as capacidades do mundo natural (e igualmente do social e econômico nas ciências humanas), noção que evoca as potências aristotélicas.

5 Para detalhes históricos da introdução dos métodos de renormalização na Eletrodinâmica Quântica, ver Schweber, 1994.

${ }_{6}$ É uma situação análoga a um experimento de dupla fenda. Apesar de os resultados mensuráveis poderem ser explicados fazendo uso da interferência de ondas (os inúmeros elementos do cálculo perturbativo no espalhamento), os resultados mensuráveis são dados pelo produto interno da sobreposição (da série). Outra analogia, esta devido ao filósofo da física Michael Redhead (1988), é com a análise de Fourier da nota emitida por um violino. Haveria sentido em falar da existência dos inúmeros harmônicos que compõem a nota?

7 Mesmo que, em abstrato, tomássemos um DF composto de infinitos ramos que representasse, em "máximo isomorfismo", a Matriz-S, a convergência desta nem sempre está bem definida. Assim, esse objeto ainda teria um caráter de idealização e papel pragmático investigativo, de acordo com a caracterização geral que fizemos de modelo científico.

8 Matematicamente, reordenar os termos de uma série, separando-a em diferentes subséries, só é justificado se ela converge absolutamente. Se isso não está provado no caso considerado, podemos considerar a aplicação do procedimento ainda mais um nível de modelização.

9 É comum, na literatura mencionada, que os autores façam referências passageiras à existência de um campo e a relação das partículas com o campo, mas sem se aprofundar na discussão e sempre dando preferência ao termo "partícula".

10 Tomando um ensino em progressão histórica de Física Clássica, MQ e TQC, essa abordagem já promoveria uma "desenvolução" do ensino de Física, por parte do ensino de TQC, para um estágio anterior a um dos objetivos educacionais do ensino de MQ: em FMC não se descreve movimentos de pontos materiais no espaço-tempo.

\section{Referências}

ADÚRIZ-BRAVO, Agustín. Teaching the Nature of Science with Scientific Narratives. Interchange, v. 45, n. 3-4, p. 167-184. nov. 2014.

ALLDAY, Jonathan. The nature of force in particle physics. Physics Education, v. 32, n. 5, p. 327-32. set. 1997.

ARIZA, Yefrin; LORENZANO, Pablo; ADÚRIZ-BRAVO, Agustín. Meta-Theoretical Contributions to the Constitution of a Model-Based Didactics of Sciences. Science \& Education, v. 25, n. 7, p. 747-773. jul. 2016. 
VAN DER BERG, Eduard; HOEKZEMA, Dick. Teaching conservation laws, symmetries and elementary particles with fast feedback. Physics Education, v. 41, n. 1, p. 47-56. jan. 2006.

CARTWRIGHT, Nancy. How the Laws of Physics Lie. Oxford University Press, 1983.

CARTWRIGHT, Nancy. The Dappled World: A Study of the Boundaries of Science. Cambridge University Press, 1999.

COLL, Richard. K., FRANCE, Bev; TAYLOR, Ian. The role of models/and analogies in science education: implications from research. International Journal of Science Education. v. 7, n. 2. p. 183-198. jan. 2005.

DANIEL, Michael. Particles, Feynman Diagrams and all that. Physics Education, v. 41, p. 119-29. mar, 2006.

DRIVER, Rosalind, LEACH, John, MILLAR, Robin; SCOTT, Phil. Young people's images of science. Buckingham, UK: Open University Press. 1996

DUNNE, Peter. Looking for consistency in the construction and use of Feynman Diagrams. Physics Education, v. 36, n. 5, p. 366-37. set. 2001.

DUSCHL. Richard. A.; GRANDY, Richard. Two Views About Explicitly Teaching Nature of Science. Science \& Education, v. 22, n. 9, p. 2109-2139. set. 2013.

DUTRA, Luiz Henrique de A. Pragmática de Modelos: natureza, estrutura e uso dos modelos científicos. Edições Loyola, 2013.

EISBERG, Robert.; RESNICK, Robert. Quantum Physics of Atoms, Molecules, Solids, Nuclei and Particles. John Wiley \& Sons. 2nd edition. 1985

FARMELO, Graham. Teaching particle physics in the open university's science foundation course. Physics Education, v. 27, p. 96-101. mar. 1992.

GILBERT, John K. Models and Modelling: routes to more authentic science education. International Journal of Science and Mathematics Education, v. 2, n. 2, p. 115-130. jun. 2004.

HOBSON, Art. Electrons as field quanta: a better way to teach quantum physics in introductory general physics courses. American Journal of Physics, v. 73, p. 630-4. jul. 2005.

HOBSON, Art. Teaching Elementary Particle Physics, part I. The Physics Teacher, v. 49, n. 1, p. 12-15. jan. 2011

HOBSON, Art There are no particles, there are only fields. American Journal of Physics, v. 81, p. 211-23. mar. 2013.

HODSON, Derek. Realçando o papel da ética e da política na educação científica: algumas considerações teóricas e práticas sobre questões sociocientíficas. In: CONRADO, D. M.; NUNES-NETO, N. Questões sociocientíficas: fundamentos, propostas de ensino e perspectivas para ações sociopolíticas [online]. Salvador: EDUFBA, 2018. p. 27-57

HOEKZEMA, Dick. et al. Conservation laws, Symmetries, and Elementary Particles. The Physics Teacher, v. 43, n. 5, p. 266-271. mai. 2005.

IRZIK, Gürol; NOLA, Robert. A family resemblance approach to the nature of science for science education. Science \& Education, v. 20, n. 7-8, p. 591-607, ago. 2011. 
JOHANSSON, K. E.; WATKINS, Peter M. Exploring the standard model of particles. Physics Education, v. 48, p. 105-14. 2013.

JONES, Goronwy Tudor. The uncertainty principle, virtual particles and real forces. Physics Education, v. 37, p. 223-233. mai. 2002

KAISER, David. Drawing Theories Apart: The Dispersion of Feynman Diagrams in Postwar Physics. University of Chicago Press, 2005.

KAISER, David. Stick-Figure Realism: Conventions, Reification and the Persistence of Feynman Diagrams, 1948-1964. Representations, n. 70, p. 49-86. 2000.

KIMBALL, Mekritt E. Understanding the Nature of Science: A Comparison of Scientists and Science Teachers. Journal of Research in Science Teaching. v. 5, p. 110-120. jun. 1967

KONTOKOSTAS, George; KALKANIS, George. Teaching electron-positron-photon interaction with hands-on Feynman diagrams. The Physics Teacher, v. 51, p. 232-3. mar. 2013.

LANCASTER, University. Higgs. Disponível em: http://ppp.lancs.ac.uk/higgs/en-GB/higgs. html?LPPPSession=1567036800030 Acesso em:03jun.2021

LEDERMAN, Norman G. Nature of Science: Past, Present, and Future. In: ABELL, S. K. LEDERMAN, N. G. (Eds). Research on Science Education,. Routledge, New York, USA. 2007. p. $831-879$

LERDERMAN, Norman G. La siempre cambiante contextualización de la naturaleza de la ciencia: documentos recientes sobre la reforma de la educación científica en los Estados Unidos y su impacto en el logro de la alfabetización científica. Enseñanza de las ciencias, v. 36, n. 2, p. 5-22, jun. 2018.

MARTINS, André Ferrer P. Natureza da Ciência no ensino de ciências: uma proposta baseada em “temas" e "questões”. Caderno Brasileiro de Ensino de Física, v. 32, n. 3, p. 703-737, dez. 2015.

MORRISON, Margaret \& MORGAN, Mary. Models as Mediators: Perspectives on Natural and Social Science. Cambridge University Press. 1999.

MOURA, Breno A. O que é natureza da Ciência e qual sua relação com a História e Filosofia da Ciência? Revista Brasileira de História da Ciência, v. 7, n. 1, p. 32-46, 2014.

ORGANTINI, Giovanni. Matter and Interactions: a particle physics perspective. Physics Education, v. 44, p. 544-50. set. 2011.

OSTERMANN, Fernanda. IPPOG-Brasil Live Stream. 2020. (2h04m14s) Disponível em: <https://www.youtube.com/watch?v=YxNSKFMLwc8>. Acesso em 16 de março de 2021.

OSTERMANN, Fernanda; MOREIRA, Marco Antônio. Uma revisão bibliográfica sobre a área de pesquisa "Física Moderna e Contemporânea no Ensino Médio". Investigação em Ensino de Ciências, v. 5, n. 1, p. 23-48. 2000.

PASCOLINI, Alessandro; PIETRONI, Massimo. Feynman diagrams as metaphors: borrowing the particle physicist's imagery for science communication purposes. Physics Education, v. 37, p. 324-8. jul. 2002.

PASSON, Oliver; ZÜGGE, Thomas, GREBE-ELLIS, Johannes. Pitfalls in the teaching of elementary particle physics. Physics Education. v. 54, p. 1-17. nov. 2018. 
PEREIRA, Alexsandro P.; OSTERMANN, Fernanda. Sobre o ensino de Física Moderna e Contemporânea: uma revisão da produção acadêmica recente. Investigações em Ensino de Ciências, v. 14 , n. 3 p. 393-420. 2009.

PEREIRA, Felipe Prado Corrêa; GURGEL, Ivã. O ensino da Natureza da Ciência como forma de resistência aos movimentos Anticiência: o realismo estrutural como contraponto ao relativismo epistêmico. Caderno Brasileiro de Ensino de Física, v. 37, n. 3, p. 1278-1319. dez. 2020.

PIETROCOLA, Maurício. Inovação Curricular e Gerenciamento de Riscos Didático-Pedagógicos: o ensino de conteúdos de Física Moderna e Contemporânea na escola média. FEUSP, São Paulo. 2010 Oct.

PIETROCOLA, Maurício. Uma crítica epistemológica sobre as bases do currículo: a interdisciplinaridade como um saber de segunda ordem. Educação, Sociedade e Culturas. v. 55, p. 31-51. dez. 2019.

PIETROCOLA, Maurício; GURGEL, Ivã (Eds). Crossing the Border of the Traditional Science Curriculum: Innovative Teaching and Learning in Basic Science Education. Sense Publishers, Netherlands. 2017.

REDHEAD, Michael. A Philosopher Looks at Quantum Field Theory. In: Philosophical Foundations of Quantum Field Theory. Ed. H. R. Brown \& R. Harré. Oxford: Clarendon Press. 1988. p. 9-23.

SALEM, Sônia. Perfil, evolução e perspectivas da pesquisa em ensino de Física no Brasil. 2012. Tese (Doutorado em Ensino de Ciências) - Programa de Pós-Graduação em Ensino de Ciências. Universidade de São Paulo, Instituto de Física/Faculdade de Educação, São Paulo, 2012.

SANTOS, Monique; MAIA, Poliana; JUSTI, Rosária. Um Modelo de Ciências para Fundamentar a Introdução de Aspectos de Natureza da Ciência em Contexto de Ensino e para Analisar tais Contextos. Revista Brasileira de Pesquisa em Educação em Ciências, p. 581-616, jul. 2020.

SCHWEBER, Silvan S. QED and the Men Who Made It: Dyson, Feynman, Schwinger and Tomonaga. Princeton University Press. 1994.

STÖLTZNER, Michael. Feynman Diagrams as Models. Springer Science+Business Media New York, v. 39, n. 2. 2017.

TERRAZZAN, Eduardo A. A inserção da física moderna e contemporânea no ensino de física na escola de $2^{\circ}$ grau. Caderno Catarinense de Ensino de Física, v. 9, n. 3, p. 209-214. jan. 1992.

VAN FRAASSEN, Bas C. Quantum Mechanics: an empiricist view. Clarendon Press, Oxford. 1991

WOITHE, Julia; WIENER, Gerfried; VAN DER VEKEN, Frederik F. Let us have coffee with the Standard Model of particle physics! Physics Education, v. 52, 034001. mar. 2017.

YOUNG, Michael F. D. The future of education in a knowledge society: The radical case for a subject-based curriculum. Journal of the Pacific Circle Consortium for Education, v. 22, n. 1. dez. 2010.

ZANETIC, João. Física Também é Cultura. 1989. Tese (Doutorado em Educação) - Programa de Pós-Graduação em Educação. Universidade de São Paulo, Faculdade de Educação, São Paulo, 1989. 